\title{
TIME OPTIMAL CONTROL OF FLEXIBLE SYSTEMS SUBJECT TO FRICTION
}

\author{
Jae-Jun Kim * $\quad$ Rajaey Kased ${ }^{\dagger}$ \\ Tarunraj Singh ${ }^{\ddagger}$ \\ State University of New York at Buffalo, Buffalo, NY 14260
}

\begin{abstract}
This paper presents a technique for the determination of timeoptimal control profiles for a rest-to-rest maneuvering multiple spring-mass-damper system, subject to Coulomb friction. A parameterization of the control input which accounts for the friction force, resulting in a linear analysis of the system is proposed. Optimality condition are examined for the control profile resulting from the parameter optimization problem. The variation of the optimal control structure as a function of final displacement is exemplified on the friction benchmark problem.
\end{abstract}

\section{INTRODUCTION}

Mechanical systems involving relative motion are always under the influence of frictional forces. Therefore, it is important to include the frictional effect for designing controllers when precise regulation of the system is required. However, friction phenomenon involves very complex processes such as pre-sliding, local memory, and frictional lag. ${ }^{1}$ Because the friction force is highly nonlinear and negatively sloped in the small velocity region, flexible systems with vibratory motion may cause stickslip. Therefore, finding a time-optimal control for such a system is a challenging problem. Time-optimal control of linear flexible structures can be found in numerous papers. ${ }^{2}$ Singh and Vadali proposed a time-delay filter which shapes the step input into bang-bang control input. ${ }^{3}$ Driessen and Sadegh formulated a mixed integer linear programming problem to design a near time-optimal controller including the Coulomb friction effect. ${ }^{4}$ However, finding an accurate optimal control profile with the mixed integer programming is computationally very expensive, and the accuracy is limited by the number of samples and convergence tolerance. In this paper, exact time-optimal control of the single input flexible system is developed where the actuated mass is also subject to Coulomb friction. The proposed development illustrates that the input-shaping/time-delay filter can be used to determine the optimal control profile for certain maneuvers. This is possible by parameterizing an equivalent input which includes the Coulomb friction effect. A new technique to solve for the switch and the final times is also presented when the stiction occurs where the input-shaping/time-delay filter cannot be used. The optimality proof is also included for the proposed technique. The numerical simulation is performed on a two-mass benchmark problem, where the Coulomb friction is acting on the first mass. The resulting controller can be applied to many applications such as hard disk drives and flexible robot arms where the friction force is acting on the pivot and the end effector position is to be regulated.

\footnotetext{
*Graduate Student: jjk@eng.buffalo.edu

† Graduate Student: kased@eng.buffalo.edu

${ }^{\ddagger}$ Associate Professor: tsingh@eng.buffalo.edu
}

\section{PROBLEM FORMULATION}

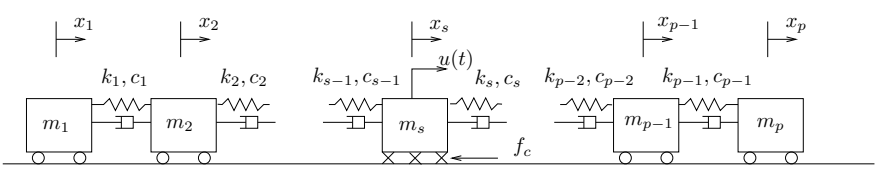

Fig. 1 General $p$ Mass-Spring System Subject to Friction

Let us consider the minimum time control problem for a general $p$ mass-spring-damper system shown in Figure 1. A single control input acts on the the $s^{t h}$ mass, which is also subject to the Coulomb friction $f_{c}$. The time optimal problem statement for a rest-to-rest maneuver is given as

$$
\begin{aligned}
& \text { minimize } \quad \int_{0}^{t_{f}} \mathrm{~d} t \quad \text { subject to } \\
& \underline{\dot{x}}(t)=A \underline{x}(t)+B\left(u(t)-f_{c} \operatorname{sign}\left(\dot{x}_{s}\right)\right) \\
& \underline{x}(0)=\underline{x}_{0}, \quad \underline{x}\left(t_{f}\right)=\underline{x}_{f} \\
& -U \leq u(t) \leq U
\end{aligned}
$$

where $A$ and $B$ are defined as

$$
A=\left[\begin{array}{cc}
0_{p \times p} & I_{p \times p} \\
-M^{-1} K & -M^{-1} C
\end{array}\right], \quad B=\left[\begin{array}{c}
0_{p \times 1} \\
M^{-1} D
\end{array}\right]
$$

$M, K$, and $C$ are the mass, stiffness, and damping matrices respectively. $D$ is a control influence vector of zeros with a nonzero value in the $s^{t h}$ entry. $f_{c}$ is the Coulomb friction coefficient which is assumed to be constant.

\section{PARAMETERIZATION OF CONTROL}

It has been previously shown by Singh and Vadali, ${ }^{3}$ that time delay filters can be used to satisfy the boundary condition of the rest-to-rest maneuvers. However, the systems that are analyzed here are nonlinear systems and the concept of poles and zeros are nonexistent. Thus the time delay filter approach cannot be used in its current form. In order to develop a time-delay filter approach, a linearizing net input is introduced as

$$
u_{n e t}(t)=u(t)-f_{c} \operatorname{sign}\left(\dot{x}_{s}\right)
$$

Since the nonlinearity of the system is taken into account with this new input, the system to be analyzed is linear with added constraints on the parameterizations which will account for the friction. The system equation shown in Equation (1) can be rewritten in linear form as

$$
\underline{\dot{x}}(t)=A \underline{x}(t)+B u_{n e t}(t)
$$

If we parameterize the linearizing input, $u_{\text {net }}(t)$, instead of the actual input, $u(t)$, then time delay filters can be used to solve the time optimal problem stated in Equation (1). Figure 2 shows a 
schematic of the thought process in developing this technique for a specific two mass spring system where the control and frictional force are acting on the first mass. The velocity profile of the first mass is assumed to be the first plot in Figure 2. The second plot in Figure 2 is the actual input to the system, assuming no singular interval, to be bang bang. The new input is parameterized and is shown as the final plot, which is the sum of actual input and friction force. For the general multiple mass spring system shown

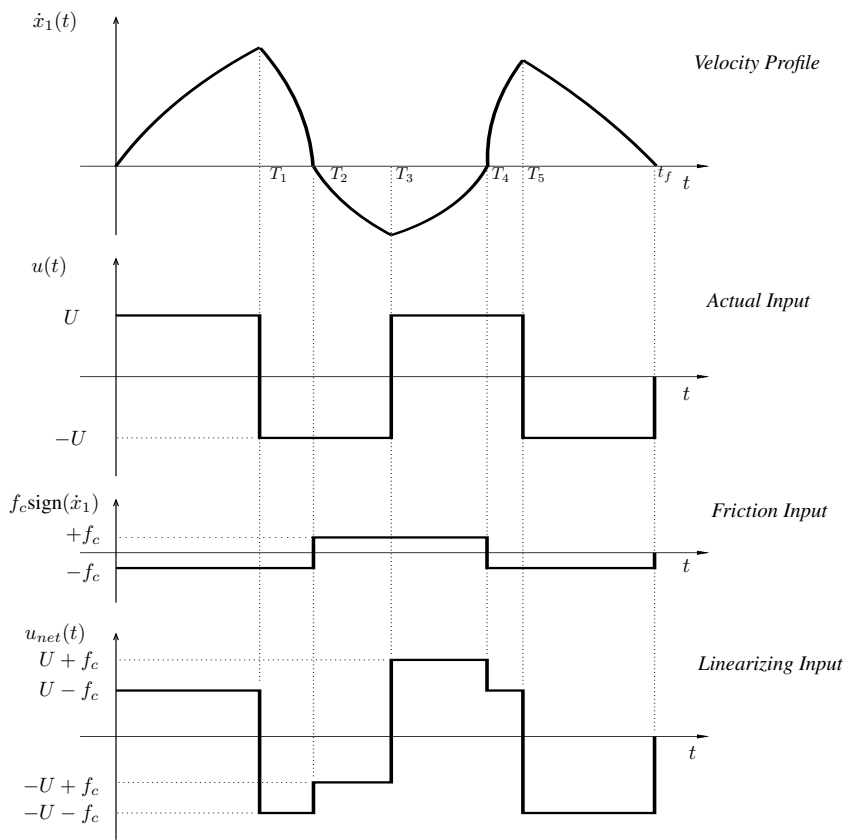

Fig. 2 Schematic of Linearizing Input Parameterizations for Two Mass Spring System.

in Figure 1, we assume that the control has $n$ switch times at the $\ell_{i}^{t h}$ time instant $(i=1 \ldots n)$ and the velocity of the $s^{t h}$ mass is assumed to change its sign $m$ times at the $k_{j}^{\text {th }}$ time instant $(j=1 \ldots m)$. For example, $\ell=[1,3,5](n=3)$ and $k=[2,4]$ $(m=2)$ for the problem in Figure 2. The time delay filter which generates the control profile when driven by a unit step input can be parameterized as

$$
\begin{aligned}
G(s) & =\left(U-f_{c}\right)+2 U \sum_{i=1}^{n}(-1)^{i} e^{-s T_{\ell_{i}}} \\
& +2 f_{c} \sum_{j=1}^{m}(-1)^{j+1} e^{-s T_{k_{j}}}+\left(U+f_{c}\right) e^{-s t_{f}}
\end{aligned}
$$

$G(s)$ must cancel out all of the poles of the system while the control input satisfies the final boundary conditions shown in Equation (1). One of the rigid body poles is cancelled due to Equation (5) having a zero at $s=0$. To cancel the other rigid body pole, the time derivative of (5) must also have a zero at $s=0$. In order to cancel the flexible mode poles, Equation (5) evaluated at $s_{\mu}=\sigma_{\mu}+i \omega_{\mu}$ should be zeros for $\mu=1 \ldots(p-1)$. The next constraint comes from ensuring that the final boundary condition on position is satisfied. This can be found by using the final value theorem. The final value theorem can be represented as

$$
x_{s}\left(t_{f}\right)=\lim _{s \rightarrow 0} G(s) G_{p}(s)
$$

where $G_{p}(s)$ is the transfer function of the $s^{t h}$ mass position output derived from Equation (4). For rest to rest maneuvers, the final position of the each mass must be the same. $L^{\prime} H o p i t a l^{\prime} s$ rule is used to solve Equation (5). The next $m$ constraints are the velocity constraints at the time when the friction sign changes, which are shown in Equation (6).

$$
v_{j}(t)=\left.\mathcal{L}^{-1}\left[G(s) G_{p}(s)\right]\right|_{T_{k_{j}}}=0, \quad j=1 \ldots m
$$

There are total of $2 p+m$ constraints with $n+m+1$ unknowns (i.e. the switch and final times). Mat lab's optimization toolbox is used to solve for the unknown switch and final times while minimizing the final time subject to the previous constraints.

\section{OPTIMALITY CONDITION}

The time optimal control problem with the new development in Section is written as

$$
\begin{aligned}
& \text { minimize } \int_{0}^{t_{f}} d t \quad \text { subject to } \\
& \quad \dot{x}^{\dot{x}}=A \underline{x}+B\left(u+(-1)^{j} f_{c}\right), \quad T_{j-1}<t<T_{j} \\
& \dot{x}_{s}\left(T_{k_{j}}\right)=0 \\
& \underline{x}(0)=\underline{x}_{0} \text { and } \underline{x}\left(t_{f}\right)=\underline{x}_{f} \\
& -U \leq u \leq U
\end{aligned}
$$

for $j=1 \ldots m$ and $T_{0}=0$. It is assumed in Equation 7 that the frictional mass starts to maneuver from rest in the positive direction with positive velocity. Equation (7) has additional interior point constraints when the velocity of the frictional body is zero compared to the initial problem statement in Equation (1). The Lagrangian of this problem can be written as ${ }^{5}$

$$
L=\underline{\nu}^{T} \underline{N}+\int_{0}^{t_{f}}\left(H-\underline{\lambda}^{T} \underline{\dot{x}}\right) d t
$$

where, Hamiltonian and interior point constraints are

$$
\begin{aligned}
& \mathbf{H}=1+\underline{\lambda}^{T}\left(A \underline{x}+B\left(u+(-1)^{j} f_{c}\right)\right), \quad T_{j-1}<t<T_{j} \\
& \underline{N}=\left[\dot{x}_{s}\left(T_{k_{1}}\right) \dot{x}_{s}\left(T_{k_{2}}\right) \ldots \dot{x}_{s}\left(T_{k_{m}}\right)\right]^{T}=\underline{0}
\end{aligned}
$$

for $j=1 \ldots m$ and the Lagrangian multipliers are defined as

$$
\nu_{j}\left\{\begin{array}{ll}
\geq 0 & t=T_{k_{j}} \\
=0 & \text { elsewhere }
\end{array} j=1 \ldots m\right.
$$

Since the Hamiltonian is not explicitly a function of time, it is equal to zero for all time $t$. As in the system shown in Equation (1), the switching function for a new problem is also given as $B^{T} \underline{\lambda}(t)$. We also know that for optimality, the switching function must cross zero at the switching times such that

$$
B^{T} \underline{\lambda}\left(T_{\ell_{i}}\right)=0, \quad i=1 \ldots n
$$

The co-state equation from the optimality condition becomes

$$
\underline{\dot{\lambda}}=-\frac{\partial \mathbf{H}}{\partial \underline{x}}=-A^{T} \lambda, \quad \text { for all time except } t=T_{k_{j}}
$$

for $j=1 \ldots m$. when $t=T_{k_{j}}$, the co-states should satisfy the following equations.

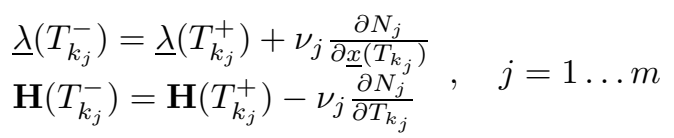


Because $\underline{N}$ is not explicitly a function of time, the Hamiltonian should be continuous such that $\mathbf{H}\left(T_{k_{j}}^{-}\right)=\mathbf{H}\left(T_{k_{j}}^{+}\right)$. Therefore, jump discontinuity in the co-state has to be chosen to satisfy the continuous Hamiltonian requirements, which can be written in the form

$$
\nu_{j} \frac{\partial N_{j}}{\partial \underline{x}\left(T_{k_{j}}\right)}=\gamma_{j} B^{T} \underline{\lambda}\left(T_{k_{j}}^{-}\right)
$$

Once $\nu_{j}$ 's are determined, we can find an expression for $\underline{\lambda}\left(T_{\ell_{i}}\right)$, in terms of the initial co-states, $\underline{\lambda}(0)$, from Equation (12) and (13). Then we can build Equation (11) in terms of $\underline{\lambda}(0)$. As an example, assume that the first time that the velocity goes to zero is $T_{k_{1}}$, then the co-states at $T_{k_{1}}^{-}$are given by equation (15).

$$
\underline{\lambda}\left(T_{k_{1}}^{-}\right)=e^{-A^{T} T_{k_{1}}^{-}} \underline{\lambda}(0)
$$

The co-states at $t=T_{k_{1}}^{+}$are given as

$$
\underline{\lambda}\left(T_{k_{1}}^{+}\right)=\left(\mathcal{I}+J_{1}\right) \underline{\lambda}\left(T_{k_{1}}^{-}\right)=\left(\mathcal{I}+J_{1}\right) \underline{\lambda} e^{-A^{T} T_{k_{1}}^{-}} \underline{\lambda}(0)
$$

where, $J_{1}$ is a $2 p \times 2 p$ matrix where $(p+s)^{t} h$ row is $-\gamma_{j} B^{T}$ and zeros for the rest, and $\mathcal{I}$ is a $2 p \times 2 p$ identity matrix. Then the costates can be integrated until the next switch with this new initial conditions. This procedure is repeated until the $n \times 2 p$ matrix $\mathcal{M}$ is formed for $n$ switch times such that

$$
\left[\begin{array}{l}
B^{T} \underline{\lambda}\left(T_{\ell_{1}}\right) \\
B^{T} \underline{\lambda}\left(T_{\ell_{2}}\right) \\
\cdots \\
B^{T} \underline{\lambda}\left(T_{\ell_{n}}\right)
\end{array}\right]=\mathcal{M} \underline{\lambda}(0)=\underline{0}
$$

$\underline{\lambda}(0)$ is found by the null space of $\mathcal{M}$ which satisfies the Hamiltonian requirements such that $\mathbf{H}(t=0)=0$. After calculating $\underline{\lambda}(0)$ from Equation (17), the co-states can then be integrated forward and the resulting switching function must cross zero at the switching time to ensure optimality.

\section{NUMERICAL EXAMPLE}

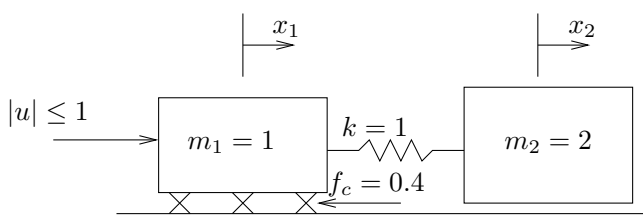

Fig. 3 Two Mass Harmonic System Subject to Coulomb Friction

The example problem considered is illustrated in Figure 3. The control input and Coulomb friction forces are acting on the first mass. The time optimal control problem can be stated as

$$
\begin{gathered}
\text { minimize } \int_{0}^{t_{f}} d t \quad \text { subject to } \\
\underline{\dot{x}}(t)=\left[\begin{array}{cccc}
0 & 0 & 1 & 0 \\
0 & 0 & 0 & 1 \\
-1 & 1 & 0 & 0 \\
0.5 & -0.5 & 0 & 0
\end{array}\right] \underline{x}(t)+\left[\begin{array}{l}
0 \\
0 \\
1 \\
0
\end{array}\right]\left(u-f_{c} \operatorname{sign}\left(x_{3}\right)\right) \\
\underline{x}(0)=\left[\begin{array}{llll}
0 & 0 & 0 & 0
\end{array}\right]^{T}, \underline{x}\left(t_{f}\right)=\left[\begin{array}{llll}
1 & 1 & 0 & 0
\end{array}\right]^{T},-1 \leq u \leq 1
\end{gathered}
$$

With the knowledge that the time optimal control profile of the linear undamped two mass spring system has 3 switches and 2 zero velocity crossings, we assume $n=3, m=2, k=\left[\begin{array}{ll}2 & 4\end{array}\right]$, $\ell=\left[\begin{array}{lll}1 & 3 & 5\end{array}\right]$. The flexible poles are given by $s= \pm \omega i$ where $\omega=\sqrt{1.5}$. The net control input is parameterized as shown in Figure 2. Then the parameter optimization problem can be stated as:

$$
\text { minimize } t_{f}
$$

subject to the switch time constraints,

$$
0 \leq T_{1} \leq T_{2} \leq T_{3} \leq T_{4} \leq T_{5} \leq t_{f}
$$

rigid body pole cancellation constraint,

$$
2 T_{1}-2 f_{c} T_{2}-2 T_{3}+2 f_{c} T_{4}+2 T_{5}-\left(1+f_{c}\right) t_{f}=0
$$

flexible body pole cancellation constraints,

$$
\begin{aligned}
& \left(1-f_{c}\right)-2 U \cos \left(\omega T_{1}\right)+2 f_{c} \cos \left(\omega T_{2}\right)+2 \cos \left(\omega T_{3}\right) \\
& -2 f_{c} \cos \left(\omega T_{4}\right)-2 \cos \left(\omega T_{5}\right)+\left(1+f_{c}\right) \cos \left(\omega t_{f}\right)=0 \\
& \quad-2 \sin \left(\omega T_{1}\right)+2 f_{c} \sin \left(\omega T_{2}\right)+2 \sin \left(\omega T_{3}\right) \\
& -2 f_{c} \sin \left(\omega T_{4}\right)-2 \sin \left(\omega T_{5}\right)+\left(1+f_{c}\right) \sin \left(\omega t_{f}\right)=0
\end{aligned}
$$

final displacement constraint,

$$
\frac{1}{4}\left(-2 T_{1}^{2}+2 f_{c} T_{2}^{2}+2 T_{3}^{2}-2 f_{c} T_{4}^{2}-2 T_{5}^{2}+\left(1+f_{c}\right) t_{f}^{2}\right)=1
$$

and first mass velocity constraints,

$$
\begin{gathered}
\frac{1}{m_{1}+m_{2}} \quad\left[\left(1-f_{c}\right)\left(\frac{m_{2}}{m_{1} \omega} \sin \left(\omega T_{2}\right)+T_{2}\right)\right. \\
\left.-2\left(\frac{m_{2}}{m_{1} \omega} \sin \left(\omega\left(T_{21}\right)\right)+\left(T_{21}\right)\right)\right]=0 \\
\frac{1}{m_{1}+m_{2}} \quad\left[\left(1-f_{c}\right)\left(\frac{m_{2}}{m_{1} \omega} \sin \left(\omega T_{4}\right)+T_{4}\right)\right. \\
-\quad 2\left(\frac{m_{2}}{m_{1} \omega} \sin \left(\omega\left(T_{41}\right)\right)+\left(T_{41}\right)\right) \\
+\quad 2 f_{c}\left(\frac{m_{2}}{m_{1} \omega} \sin \left(\omega\left(T_{42}\right)\right)+\left(T_{42}\right)\right) \\
\left.+\quad 2\left(\frac{m_{2}}{m_{1} \omega} \sin \left(\omega\left(T_{43}\right)\right)+\left(T_{43}\right)\right)\right]=0
\end{gathered}
$$

where $T_{a b}$ stands for $T_{a}-T_{b}$ in Equations (25) and (26). The constrained nonlinear optimizer of MatLab was used to solve this problem. The time-delay filter is found to be

$$
\begin{aligned}
G(s) & =0.6-2 e^{-1.8164 s}+0.8 e^{-2.1177 s}+2 e^{-3.1498 s} \\
& -0.8 e^{-3.5918 s}-2 e^{-4.4047 s}+1.4 e^{-5.2298 s}
\end{aligned}
$$

Co-states and switching curve can be computed with the resulting control profile to determine the optimality. The Hamiltonian of the problem is

$$
\mathbf{H}= \begin{cases}1+\lambda^{T}\left(A \underline{x}+B\left(u-f_{c}\right)\right) & 0<t<T_{2} \\ 1+\lambda^{T}\left(A \underline{x}+B\left(u+f_{c}\right)\right) & T_{2}<t<T_{4} \\ 1+\lambda^{T}\left(A \underline{x}+B\left(u-f_{c}\right)\right) & T_{4}<t<t_{f}\end{cases}
$$

and the interior point constraints are

$$
N_{1}=\dot{x}_{1}\left(T_{2}\right)=0 \quad \text { and } \quad N_{2}=\dot{x}_{1}\left(T_{4}\right)=0 .
$$


The Hamiltonian should be continuous such that $\mathbf{H}\left(T_{2}^{+}\right)=$ $\mathbf{H}\left(T_{2}^{-}\right)$and $\mathbf{H}\left(T_{4}^{+}\right)=\mathbf{H}\left(T_{4}^{-}\right)$. The switching function is $B^{T} \underline{\lambda}=\lambda_{3}$ and there is a discontinuity only in $\lambda_{3}$. The continuous Hamiltonian requirement yields

$$
\begin{aligned}
& 1+\left[\lambda_{1}\left(T_{2}\right) \lambda_{2}\left(T_{2}\right) \lambda_{3}\left(T_{2}^{-}\right) \lambda_{4}\left(T_{2}\right)\right]\left(A \underline{x}\left(T_{2}\right)+B\left(-1-f_{c}\right)\right) \\
& \left.=1+\left[\lambda_{1}\left(T_{2}\right) \lambda_{2}\left(T_{2}\right) \lambda_{3}\left(T_{2}^{+}\right) \lambda_{4}\left(T_{2}\right)\right] \overline{(A \underline{x}}\left(T_{2}\right)+B\left(-1+f_{c}\right)\right) \\
& 1+\left[\lambda_{1}\left(T_{4}\right) \lambda_{2}\left(T_{4}\right) \lambda_{3}\left(T_{4}^{-}\right) \lambda_{4}\left(T_{4}\right)\right]\left(A \underline{x}\left(T_{4}\right)+B\left(1+f_{c}\right)\right) \\
& =1+\left[\lambda_{1}\left(T_{4}\right) \lambda_{2}\left(T_{4}\right) \lambda_{3}\left(T_{4}^{+}\right) \lambda_{4}\left(T_{4}\right)\right]\left(A \underline{x}\left(T_{4}\right)+B\left(1-f_{c}\right)\right)
\end{aligned}
$$

Assuming $\lambda_{3}\left(T_{2}^{+}\right)=\lambda_{3}\left(T_{2}^{-}\right)+\gamma_{1} \lambda_{3}\left(T_{2}^{-}\right)$and $\lambda_{3}\left(T_{4}^{+}\right)=$ $\lambda_{3}\left(T_{4}^{-}\right)+\gamma_{2} \lambda_{3}\left(T_{4}^{-}\right), \gamma_{1}$ and $\gamma_{2}$ are found to be

$$
\begin{aligned}
\gamma_{1} & =\frac{-2 f_{c}}{-1+f_{c}-k\left(x_{1}\left(T_{2}\right)-x_{2}\left(T_{2}\right)\right)} \\
\gamma_{2} & =\frac{2 f_{c}}{1-f_{c}-k\left(x_{1}\left(T_{4}\right)-x_{2}\left(T_{4}\right)\right)}
\end{aligned}
$$

Now the co-states at the switch times are found in terms of $\underline{\lambda}(0)$ such that

$$
\begin{aligned}
& \underline{\lambda}\left(T_{1}\right)=e^{-A^{T} T_{1}} \underline{\lambda}(0) \\
& \underline{\lambda}\left(T_{2}^{-}\right)=e^{-A^{T}\left(T_{2}-T_{1}\right)} \underline{\lambda}\left(T_{1}\right) \\
& \underline{\lambda}\left(T_{2}^{+}\right)=\left(I+J_{1}\right) \underline{\lambda}\left(T_{2}^{-}\right) \\
& \underline{\lambda}\left(T_{3}\right)=e^{-A^{T}\left(T_{3}-T_{2}\right)} \underline{\lambda}\left(T_{2}^{+}\right) \\
& \underline{\lambda}\left(T_{4}^{-}\right)=e^{-A^{T}\left(T_{4}-T_{3}\right)} \underline{\lambda}\left(T_{3}\right) \\
& \underline{\lambda}\left(T_{4}^{+}\right)=\left(I+J_{2}\right) \underline{\lambda}\left(T_{4}^{-}\right) \\
& \underline{\lambda}\left(T_{5}\right)=e^{-A^{T}\left(T_{5}-T_{4}\right)} \underline{\lambda}\left(T_{4}^{+}\right)
\end{aligned}
$$

where, $J_{1}$ and $J_{2}$ is defined as

$$
J_{1}=\left[\begin{array}{cccc}
0 & 0 & 0 & 0 \\
0 & 0 & 0 & 0 \\
0 & 0 & \gamma_{1} & 0 \\
0 & 0 & 0 & 0
\end{array}\right] \text { and } J_{2}=\left[\begin{array}{cccc}
0 & 0 & 0 & 0 \\
0 & 0 & 0 & 0 \\
0 & 0 & \gamma_{2} & 0 \\
0 & 0 & 0 & 0
\end{array}\right]
$$

$\mathcal{M}$ is now given to satisfy $\mathcal{M} \underline{\lambda}(0)=\underline{0}$ when $t=\left[T_{1}, T_{3}, T_{5}\right]$. The initial co-states are found to satisfy $\mathcal{M} \underline{\lambda}(0)=\underline{0}$ and $\mathbf{H}(t=$ $0)=0$ such that

$$
\underline{\lambda}(0)=-\frac{\operatorname{Null}(\mathcal{M})}{[\operatorname{Null}(\mathcal{M})]^{T} B\left(1-f_{c}\right)}
$$

where, $\operatorname{Null}(\mathcal{M})$ is the null space of $\mathcal{M}$. The linearizing input, as well as the switching curve is shown in figure 4 for the example problem. The actual input is found by subtracting the friction force from the net input.

\section{VARIATION OF CONTROL STRUCTURE}

In the previous example, the velocity of the first mass is zero at $T_{2}$ and $T_{4}$. As the final position increases for the previous example, the time gap between $T_{3}$ and $T_{4}$ becomes small until they coincide. The control profile for this transition indicate that the velocity crossing coincides with the control switch which will result in a four switch control profile. The transition displacement for this example is $d \approx 3.4$. After the transition displacement, going back to original profile does not provide a feasible solution. Also, keeping the four switch transition profile after the transition displacement, creates an over-constrained problem. Therefore,

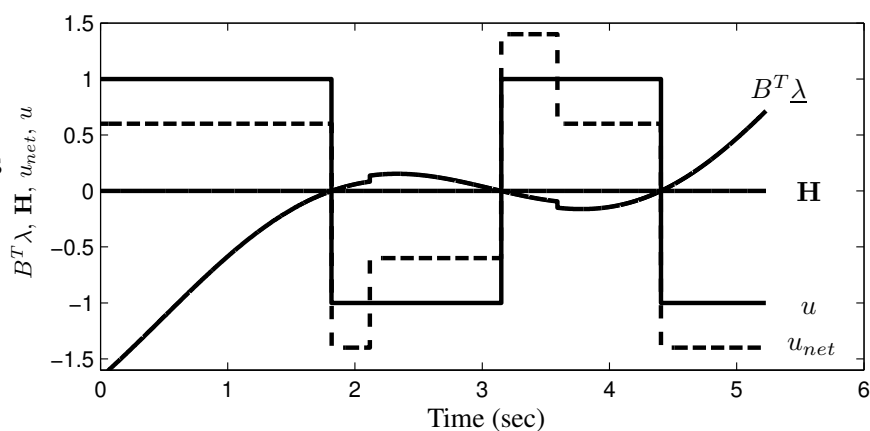

Fig. 4 Control Input, Switching Function, and Hamiltonian

the velocity of the first mass should cross zero before the new control switch activates. This implies that now $T_{3}$ is the time when the velocity is zero and $T_{4}$ is the time when the control switches. At $T_{3}^{+}$, the velocity becomes positive and therefore friction force becomes $-f_{c}$. However, the linearizing input with the new friction value drives the first mass to the negative direction if the net input is less than zero. Once the first mass crosses the zero velocity, the net input changes its sign again because of the change in friction value to $+f_{c}$. This "chattering" is equiva-

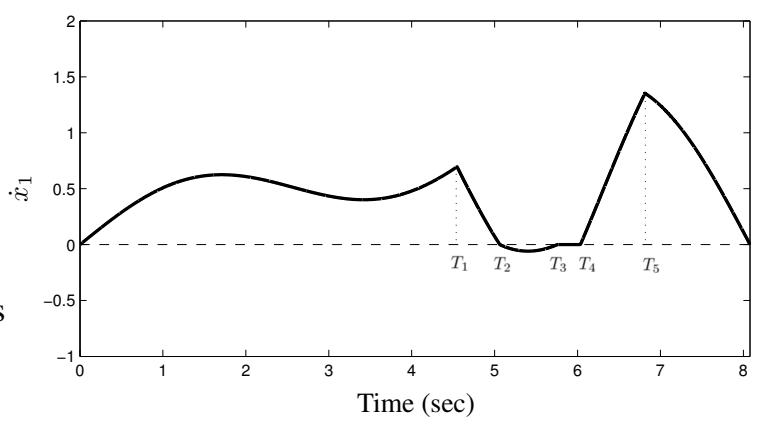

Fig. 5 Velocity Profile After Transition Displacement

lent to the stiction caused by the hard nonlinearities in the friction model and it will continue until the sum of all the forces to the frictional body overcomes the friction force. The velocity of the first mass for this chattering region becomes zero, which will be the additional constraints to the problem. For the example problem, the predicted velocity profile of the first mass is plotted in Figure 5 showing that the velocity of the first mass is zero until the new switch actuates. With the velocity profile in Figure 5, the new optimal control problem with state equality constraints can be formulated as ${ }^{5}$

$$
\begin{aligned}
& \text { minimize } \quad \int_{0}^{t_{f}} d t \quad \text { subject to } \\
& \underline{\dot{x}}=A \underline{x}+B\left(u-f_{c}\right), \quad 0<t<T_{2} \\
& \underline{\dot{x}}=A \underline{x}+B\left(u+f_{c}\right), \quad T_{2}<t<T_{3} \\
& \underline{\dot{x}}=A \underline{x}+B u, \quad T_{3}<t<T_{4} \\
& \underline{\dot{x}}=A \underline{x}+B\left(u-f_{c}\right), \quad T_{4}<t<t_{f} \\
& \ddot{x}_{1}=\frac{k}{m_{1}}\left(x_{2}-x_{1}\right)+u=0, \quad T_{3}<t<T_{4} \\
& \dot{x}_{1}\left(T_{2}\right)=\dot{x}_{1}\left(T_{3}\right)=0 \\
& \underline{x}(0)=\underline{x}_{0} \text { and } \underline{x}\left(t_{f}\right)=\underline{x}_{f} \\
& -1 \leq u \leq 1
\end{aligned}
$$


Because the friction force is zero for the zero velocity, the control for $T_{3}<t<T_{4}$ should satisfy the equality constraint in Equation 36 such that

$$
u=\frac{k}{m_{1}}\left(x_{1}-x_{2}\right), \quad T_{3}<t<T_{4}
$$

Therefore, the control profile can be parameterized from the predicted velocity profile including the state constraint effect during the chattering interval. The time-delay filter cannot be used for this profile because of the feedback control effect in the chattering interval which alters the system equation. However, the control profile can be used to integrate the system forward in time using the given initial guesses of the switch times to obtain the states at the switch and final times.

$$
\begin{aligned}
& \underline{x}\left(T_{1}\right)=e^{A T_{1}} \underline{x}_{0}+\int_{0}^{T_{1}} e^{A(t-\tau)} B\left(1-f_{c}\right) d \tau \\
& \underline{x}\left(T_{2}\right)=e^{A\left(T_{2}-T_{1}\right)} \underline{x}\left(T_{1}\right)+\int_{T_{1}}^{T_{2}} e^{A(t-\tau)} B\left(-1-f_{c}\right) d \tau \\
& \underline{x}\left(T_{3}\right)=e^{A\left(T_{3}-T_{2}\right)} \underline{x}\left(T_{2}\right)+\int_{T_{2}}^{T_{3}} e^{A(t-\tau)} B\left(-1+f_{c}\right) d \tau \\
& \underline{x}\left(T_{4}\right)=e^{A^{c}\left(T_{4}-T_{3}\right)} \underline{x}\left(T_{3}\right) \\
& \underline{x}\left(T_{5}\right)=e^{A\left(T_{5}-T_{4}\right)} \underline{x}\left(T_{4}\right)+\int_{T_{4}}^{T_{5}} e^{A(t-\tau)} B\left(1-f_{c}\right)(\tau) d \tau \\
& \underline{x}\left(t_{f}\right)=e^{A\left(T_{6}-T_{5}\right)} \underline{x}\left(T_{5}\right)+\int_{T_{5}}^{T_{6}} e^{A(t-\tau)} B\left(-1-f_{c}\right)(\tau) d \tau
\end{aligned}
$$

where, $A^{c}$ is the closed loop system equation in the interval at $t \in\left[T_{3}, T_{4}\right]$ using Equation 37. For the computational accuracy and convenience, it is very useful to use the following property (van Loan identity) to compute the states at the switch times.

$$
\exp \left(\left[\begin{array}{cc}
A & B \\
0 & 0
\end{array}\right] t\right)=\left[\begin{array}{cc}
e^{A t} & \int_{0}^{t} e^{A(t-\tau)} B d \tau \\
0 & 1
\end{array}\right]
$$

Now the problem can be solved for switch times numerically by minimizing the final time $T_{6}$ with the constraints

$$
\begin{aligned}
& \dot{x}_{1}\left(T_{2}\right)=0, \quad \dot{x}_{1}\left(T_{3}\right)=0 \\
& \underline{x}\left(t_{f}\right)=\left[\begin{array}{llll}
1 & 1 & 0 & 0
\end{array}\right]^{T}
\end{aligned}
$$

The resulting control profile is used to verify the optimality of the control input by inspecting the optimality conditions. The lagrangian for this problem can be formulated $\mathrm{as}^{5}$

$$
L=\psi N_{1}+\pi N_{2}+\int_{t_{0}}^{t_{f}}\left(\mathbf{H}-\lambda^{T} \underline{\dot{x}}\right) d t
$$

where Hamiltonian and constraints are

$$
\begin{array}{lc}
\mathbf{H}=1+\lambda^{T}\left(A \underline{x}+B\left(u-f_{c}\right)\right), & 0<t<T_{2} \\
\mathbf{H}=1+\lambda^{T}\left(A \underline{x}+B\left(u+f_{c}\right)\right), & T_{2}<t<T_{3} \\
\mathbf{H}=1+\lambda^{T}(A \underline{x}+B u)+\mu C, & T_{3}<t<T_{4} \\
\mathbf{H}=1+\lambda^{T}\left(A \underline{x}+B\left(u-f_{c}\right)\right), & T_{4}<t<t_{f} \\
N_{1}=\dot{x}_{1}\left(T_{2}\right)=0 & \\
N_{2}=\dot{x}_{1}\left(T_{3}\right)=0 \\
C=\ddot{x}_{1}(t)=k\left(x_{2}-x_{1}\right)+u=0
\end{array}
$$

and the Lagrangian multipliers are defined as

$$
\begin{gathered}
\psi\left\{\begin{array} { l l } 
{ \geq 0 } & { t = T _ { 2 } } \\
{ = 0 } & { \text { elsewhere } }
\end{array} \quad \text { and } \pi \left\{\begin{array}{ll}
\geq 0 & t=T_{3} \\
=0 & \text { elsewhere }
\end{array}\right.\right. \\
\mu \begin{cases}\geq 0 & t \in\left[T_{3}, T_{4}\right] \\
=0 & \text { elsewhere }\end{cases}
\end{gathered}
$$

The input $u$ is considered to be within the saturation limit for $T_{3}<t<T_{4}$ because of the stiction assumption with $u=-1$. Then, $\mu$ can be found from the following relationship.

$$
\frac{\partial \mathbf{H}}{\partial u}=0=B^{T} \underline{\lambda}+\mu \quad \text { or } \quad \mu=-B^{T} \underline{\lambda}, \quad T_{3}<t<T_{4}
$$

Therefore, the co-state equation is found from Equation 45 and the necessary optimality condition.

$$
\underline{\dot{\lambda}}=-\frac{\partial \mathbf{H}}{\partial \underline{x}}=\left\{\begin{array}{c}
\left(-A^{T}+\frac{\partial C}{\partial \underline{x}} B^{T}\right) \underline{\lambda} \quad T_{3}<t<T_{4} \\
-A^{T} \underline{\lambda} \quad \text { elsewhere }\left(t \neq T_{2}, t \neq T_{3}\right)
\end{array}\right.
$$

At the interior points, $t=T_{2}$ and $t=T_{3}$, the co-states becomes discontinuous and are defined by the following equations.

$$
\begin{aligned}
& \lambda\left(T_{2}^{-}\right)=\lambda\left(T_{2}^{+}\right)+\psi \frac{\partial N_{1}}{\partial x\left(T_{2}\right)}, \lambda\left(T_{3}^{-}\right)=\lambda\left(T_{3}^{+}\right)+\pi \frac{\partial N_{2}}{\partial x\left(T_{3}\right)} \\
& \mathbf{H}\left(T_{2}^{-}\right)=\mathbf{H}\left(T_{2}^{+}\right)-\psi \frac{\partial N_{1}}{\partial T_{2}}, \mathbf{H}\left(T_{3}^{-}\right)=\mathbf{H}\left(T_{3}^{+}\right)-\pi \frac{\partial N_{2}}{\partial T_{3}}
\end{aligned}
$$

Because the interior point constraints are not explicitly functions of time, the Hamiltonian is continuous such that $\mathbf{H}\left(T_{2}^{-}\right)=$ $\mathbf{H}\left(T_{2}^{+}\right)$and $\mathbf{H}\left(T_{3}^{-}\right)=\mathbf{H}\left(T_{3}^{+}\right)$. Therefore, $\psi$ and $\pi$ in Equation 47 are chosen to satisfy the continuous Hamiltonian requirement which will yield the following equations

$$
\gamma_{1}=\frac{-2 f_{c}}{-1+f_{c}-k\left(x_{1}\left(T_{2}\right)-x_{2}\left(T_{2}\right)\right)}, \quad \lambda_{3}\left(T_{3}^{-}\right)=0
$$

where, $\gamma_{1}$ satisfies $\lambda_{3}\left(T_{2}^{+}\right)=\lambda_{3}\left(T_{2}^{-}\right)+\gamma_{1} \lambda_{3}\left(T_{2}^{-}\right)$. Co-states can be integrated forward in time with the initial $\lambda(0)$ using Equation 46 and 48 .

$$
\begin{aligned}
& \underline{\lambda}\left(T_{1}\right)=e^{-A^{T} T_{1}} \underline{\lambda}(0) \\
& \underline{\lambda}\left(T_{2}^{-}\right)=e^{-A^{T}\left(T_{2}-T_{1}\right)} \underline{\lambda}\left(T_{1}\right) \\
& \underline{\lambda}\left(T_{2}^{+}\right)=\left(I+J_{1}\right) \underline{\lambda}\left(T_{2}^{-}\right) \\
& \underline{\lambda}\left(T_{3}^{-}\right)=e^{-A^{T}\left(T_{3}-T_{2}\right)} \underline{\lambda}\left(T_{2}^{+}\right) \\
& \underline{\lambda}\left(T_{3}^{+}\right)=\left(I+J_{2}\right) \underline{\lambda}\left(T_{3}^{-}\right) \\
& \underline{\lambda}\left(T_{4}\right)=e^{\left(-A^{T}+\frac{\partial C}{\partial \underline{x}}\right)\left(T_{4}-T_{3}\right)} \underline{\lambda}\left(T_{3}^{+}\right) \\
& \underline{\lambda}\left(T_{5}\right)=e^{-A^{T}\left(T_{5}-T_{4}\right)} \underline{\lambda}\left(T_{4}\right)
\end{aligned}
$$

Where, $I$ is an $4 \times 4$ Identity Matrix and

$$
J_{1}=\left[\begin{array}{cccc}
0 & 0 & 0 & 0 \\
0 & 0 & 0 & 0 \\
0 & 0 & \gamma_{1} & 0 \\
0 & 0 & 0 & 0
\end{array}\right], \quad J_{2}=\left[\begin{array}{cccc}
0 & 0 & 0 & 0 \\
0 & 0 & 0 & 0 \\
0 & 0 & \infty & 0 \\
0 & 0 & 0 & 0
\end{array}\right]
$$

Because $\underline{\lambda}\left(T_{3}^{-}\right)$should be zero, infinite value has to be multiplied to $\underline{\lambda}\left(T_{3}^{-}\right)$in $J_{2}$ to have finite jump discontinuities in the co-states. For numerical computations, a large number is used in $J_{2}$ instead of $\infty$. Since the switching curve should cross zero at the actual 
switch times, $T_{1}, T_{4}$, and $T_{5}, \mathcal{M}$ can be determined such that $\mathcal{M} \underline{\lambda}(0)=\underline{0}$. Then, $\underline{\lambda}(0)$ is selected to satisfy Hamiltonian requirement, $H(t=0)=0$, such that

$$
\underline{\lambda}(0)=-\frac{\operatorname{Null}(P)}{[\operatorname{Null}(P)]^{T} B\left(1-f_{c}\right)}
$$

In Figure 6, the linearizing and actual control input along with the switching curve is plotted. The actual input during the stiction violates the control input limits, however, it is possible to maintain the control to be -1 and let the available friction force the system to stick. Then the actual control profile becomes three-switch bang-bang. The resulting control input satisfies the necessary optimality conditions as shown in Figure 6.

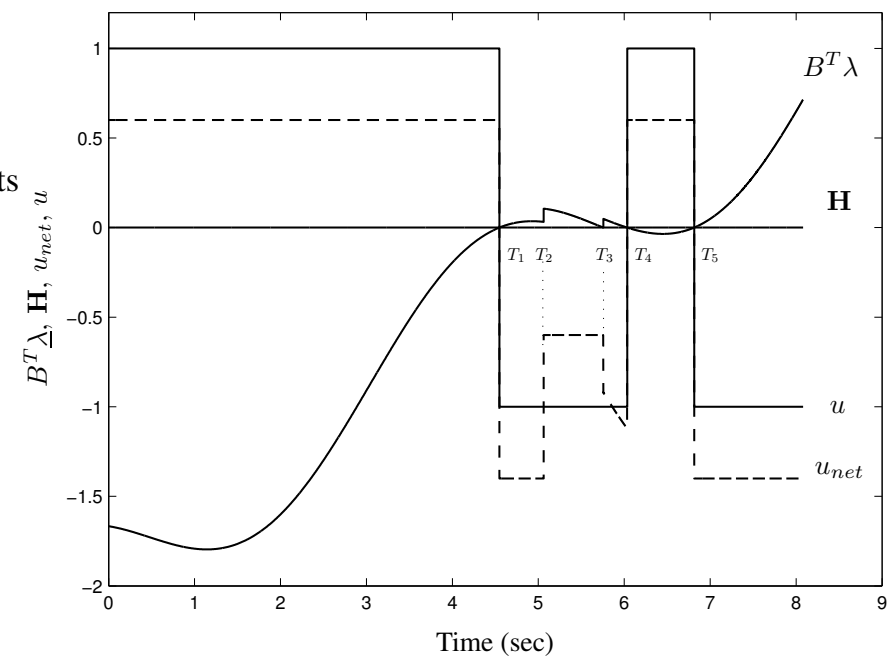

Fig. 6 Linearizing Control Input, Switching Function, and Hamiltonian

As the final displacement is further increased, the profile of the control shows further transitions. The control profile can be solved similarly as in the previous development. The summary of the control input transition profile for the example problem is shown in Figure 7. The actual control switch times are plotted
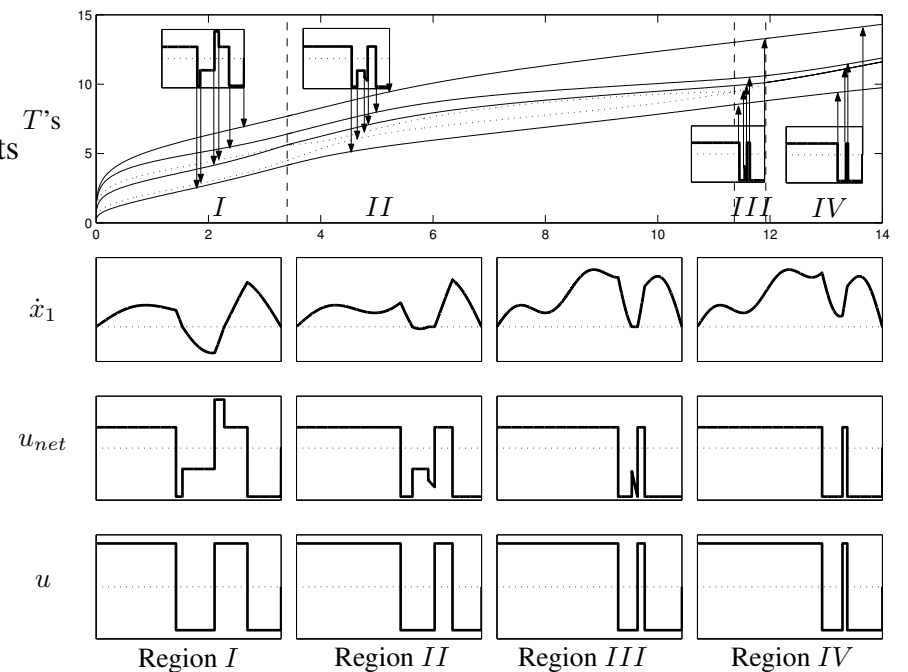

Fig. 7 Switch Time vs. Displacement with Velocity and Control Input Profiles

with the solid line as a function of final displacement showing that the control switches are smooth curves for final displacement changes. The dotted line in Figure 7 denotes the velocity switches.

\section{CONCLUSION}

It is shown that the linearizing input approach provides the time optimal control solution of the multiple mass spring damper system under the assumption of correct parametrization of the control input. The frictional benchmark problem results in a timeoptimal three-switch bang-bang control profile. The linearizing input parametrization technique can also extended to systems where one or more mass is under frictional forces. This will require a correct knowledge of velocity profiles of all the masses subject to the friction.

\section{References}

${ }^{1}$ Pierre Dupon Brian Armstrong-Helouvry and Carlos Canudas De Wit. Survey of models, analysis tools and compensation methods for the control of machines with friction. Automatica, 30(7):1083-1138, 1994.

${ }^{2}$ S. L. Scrivener and R. C. Thompson. Survey of time-optimal attitude maneuvers. Journal of Guidance, Control, and Dynamics, 17(2):225-233, March-April 1994.

${ }^{3}$ T. Singh and S. R. Vadali. Robust time-optimal control: Frequency domain approach. Journal of Guidance, Control, and Dynamics, 17(2):346-353, MarchApril 1994.

${ }^{4}$ Brian J. Driessen and Nader Sadegh. Minimum-time control of systems with coulomb friction: near global optima via mixed integer linear programming. $O p$ timal Control Applications and Methods, 22(2):51-62, July 2001.

${ }^{5}$ Jr. Arthur E. Bryson and Yu-Chi Ho. Applied Optimal Control, chapter 3. Hemisphere Publishing Corporation, 1975. 